\title{
Métodos de conservação de cadáveres humanos utilizados nas faculdades de medicina do Brasil
}

\author{
Human corpses storage methods used in medical brazilians colleges
}

\author{
Guilherme Rodrigues da Silva ${ }^{1}$, Pedro Olímpio Barros Cavalcante Cortez ${ }^{1}$, Isabela de Sousa \\ Leal Lopes ${ }^{1}$, Bruna de Alcobaça Castelo Branco Teixeira ${ }^{1}$, Noelia Maria de Sousa Leal ${ }^{2}$
}

Silva GR, Cortez POB, Lopes ISL, Teixeira BACB, Leal NMS. Métodos de conservação de cadáveres humanos utilizados nas faculdades de medicina do Brasil / Human corpses storage methods used in medical brazilians colleges. Rev Med (São Paulo). 2016 out.-dez.;95(4):156-61.

RESUMO: Introdução: Anatomia é a ciência das estruturas corporais e das relações entre essas estruturas. Uma das metodologias mais antigas para o estudo da Anatomia é a utilização de cadáveres humanos. Diante da dificuldade de aquisição de novos cadáveres nos dias atuais, a conservação dos cadáveres existentes se reveste em um ponto crucial no ensino da Anatomia Humana. Objetivo: Analisar os métodos de conservação de cadáveres humanos mais utilizados nas escolas de Medicina do Brasil. Forma de estudo: Descritivo com abordagem quantitativa. Métodos: A coleta de dados foi realizada através do envio de um questionário online para o endereço eletrônico dos professores responsáveis pela disciplina de Anatomia Humana das 242 faculdades de Medicina do Brasil. Desse universo, foram obtidas 81 respostas. Os dados obtidos foram armazenados e analisados através dos programas GoogleForms ${ }^{\circledR}$ e Microsoft Excel $\AA$. Resultado: Os dados obtidos evidenciaram que $96 \%$ das Faculdades de Medicina do Brasil utilizam cadáveres humanos nas aulas práticas de Anatomia, das quais $83,3 \%$ fazem uso de formolização e $56,4 \%$ glicerinação. Constatou-se que a formolização predomina em todas as regiões do país, exceto na região Centro-Oeste. $60,7 \%$ das faculdades que utilizam a formolização pretendem mudar o método de conservação, especialmente em virtude da insalubridade do formol. Conclusão: Através dos resultados obtidos na presente pesquisa, conclui-se que a utilização de cadáveres humanos no ensino de Anatomia é adotada pela grande maioria das faculdades brasileiras de Medicina. O método mais utilizado na conservação das peças cadavéricas ainda é a formolização, seguido da glicerinização.

Descritores: Anatomia; Cadáver; Formaldeído; Glicerol; Escolas médicas; Brasil.

\begin{abstract}
Introduction: Anatomy is the science of body structures and the relations between their structures. One of the oldest methods for the study of anatomy is the use of human cadavers. Given the current difficulty for acquisition of new dead bodies and their importance in the medical education, the conservation of in use cadavers is considered as a crucial point in the teaching of anatomy. Objective: To evaluate the conservation methods of human cadavers used in medical schools in Brazil. Study approach: Descriptive with quantitative approach. Methods: Data collection was carried out by sending an online questionnaire to the email address of those responsible for the Anatomy discipline of 242 Brazilian medical schools. 81 of these institutions answered the research. The data were stored and analyzed by using the programs GoogleForms ${ }^{\circledR}$ and Microsoft Excel ${ }^{\circledR}$. Results: The data showed that $96 \%$ of medical schools makes use of human cadavers in the practical lessons of anatomy. Of that number, $83.33 \%$ makes use of formaldehyde and $56.41 \%$ of Glycerinating method. The formaldehyde is used for the most of the Brazilians states, except for West Middle of the country. $60,7 \%$ of the universities the uses formaldehyde intend to change the method of conservation because of insalubrity of the formaldehyde. Conclusion: The use of human dead bodies in anatomy teaching proved to be of great importance because its use is adopted for a significant part of Brazilian medical schools. The method most widely used in the conservation of cadavers still is formalin fixation, followed by glycerinating.
\end{abstract}

Keywords: Anatomy; Cadaver; Formaldehyde; Glycerol; Schools, medical; Brazil.

1. Acadêmico de Medicina da Faculdade Integral Diferencial - FACID | DEVRY. E-mails: guilhermersm21@gmail.com, pedro. obcavalcante@gmail.com, isabela_leall@hotmail.com,bruna_teixeira@hotmail.com.

2. Professora Doutora de Anatomia Hümana da Faculdade Integral Diferencial - FACID $\mid$ DEVRY e da Universidade Federal do Piaú (UFPI). E-mail: noeliamsleal@gmail.com.

Autor para correspondência: Guilherme Rodrigues da Silva. E-mail: guilhermersm21@gmail.com. 


\section{INTRODUÇÃO}

termo Anatomia é empregado para designar o ramo da ciência que se ocupa do estudo da morfologia, estrutura e arquitetura do organismo vivo. A Anatomia Humana estuda as estruturas corporais e suas relações no ser humano ${ }^{1}$.

AAnatomia Humana é de fundamental importância na área médica, pois o conhecimento das estruturas e órgãos que compõem o corpo humano, assim como a função e localização de cada um, são indispensáveis para a prática clínica, diagnóstica e cirúrgica. Uma das metodologias mais antigas no estudo da Anatomia é a utilização de cadáveres humanos, que se iniciou sistematicamente no final da Idade Média e perdura até hoje nas faculdades ${ }^{2}$.

A prática anatômica, especialmente para o estudante de Medicina, funciona como uma espécie de ritual de iniciação acadêmica. É ali, diante do cadáver, que o estudante começa a se sentir médico. O cadáver é, por assim dizer, o primeiro paciente desse futuro médico ${ }^{3}$.

Diante da dificuldade de aquisição de novos cadáveres humanos nos dias atuais, a conservação dos cadáveres existentes se reveste em um ponto crucial no ensino da Anatomia.

No século XIX, foi descoberto o fixador que veio a tornar-se o ícone de conservação para as peças anatômicas, o formol ou formaldeído. A técnica por fixação e conservação através do formaldeído ainda é bastante utilizada nos dias atuais em virtude do seu baixo custo, rápida penetração tecidual e conservação por muitos anos. Entretanto, traz como desvantagem um odor forte que irrita as mucosas e que se tornou característico dos laboratórios de Anatomia, além de ser um produto volátil e tóxico ${ }^{4,5,6}$.

Diversas outras técnicas de preservação foram surgindo ao longo dos tempos. A utilização da glicerina por Karl Schelle, em 1779, representou um grande impulso na preparação de peças anatômicas, sendo aplicada por Giacomini e por Laskowski na conservação de cadáveres dos anfiteatros de Anatomia. A glicerina é um líquido claro, incolor, viscoso, com capacidade de desidratar as estruturas, a qual se atribui a sua ação antisséptica ${ }^{4,6}$. A desidratação obtida com a glicerina não altera a concentração iônica das células, o que mantém a integridade celular. Isso traz como vantagens a redução da antigenicidade dos tecidos conservados, facilidade no seu manuseio, e a possibilidade de manutenção das peças em ambiente $\operatorname{seco}^{4,6}$.

No final do século XX surgiu a plastinação, uma técnica que substitui a água e os lipídios dos tecidos corporais por um polímero. Essa técnica mantém a estrutura e características originais da peça, não apresenta odor e é de fácil conservação. Entretanto, o custo é elevado e requer um maior tempo de preparo, levando meses para finalizar o procedimento ${ }^{7}$.
Atualmente, com o grande desenvolvimento da tecnologia, diversos softwares e peças artificias estão sendo criados com atributos cada vez melhores. Porém, a tecnologia moderna ainda não foi capaz de criar nada que substituísse o uso do cadáver nos laboratórios de forma satisfatória, já que as peças artificiais não substituem com fidedignidade as características do cadáver ${ }^{3,8-12}$.

O objetivo do presente estudo é analisar os métodos de conservação de cadáveres humanos utilizados nas escolas de Medicina do Brasil.

\section{METODOLOGIA}

A presente pesquisa trata-se de um estudo de caráter exploratório-descritivo. O universo da'pesquisa foram as 242 faculdades brasileiras de Medicina que estavam em atividade no ano de 2015, cujos nomes e localizações foram obtidos através do endereço eletrônico do Ministério da Educação.

Uma vez que a pesquisa envolveu seres humanos, de acordo com a Resolução 466/12 do Conselho Nacional de Saúde, o presente projeto foi submetido ao Comitê de Ética em Pesquisa da Faculdade Integral Diferencial - FACID/DeVry, com o número de aprovação 42202914.6.0000.5211. Os docentes que concordaram em participar da pesquisa assinaram o Termo de Consentimento Livre e Esclarecido (TCLE).

A coleta de dados foi realizada através do envio de um questionário online para o endereço eletrônico dos responsáveis pela disciplina de Anatomia Humana de cada instituição. O questionário, produzido pela ferramenta GoogleForms ${ }^{\circledR}$, continha a identificação e caracterização da instituição, perguntas sobre a utilização de cadáveres humanos nas aulas práticas de Anatomia, métodos de conservação dos cadáveres, além de questões sobre a satisfação do professor quanto ao método utilizado pela instituição. Nos casos de não recebimento de resposta após o prazo de 15 dias, o questionário foi reenviado aos respectivos professores. Ao final foram obtidas 81 respostas das faculdades de Medicina do Brasil.

Os dados obtidos foram organizados e analisados através dos programas GoogleForms ${ }^{\circledR}$ e Microsoft Excel ${ }^{\circledR}$, e os resultados foram apresentados na forma de gráficos.

\section{RESULTADOS}

Foram obtidas respostas de 81 Faculdades de Medicina de todas as regiões brasileiras, sendo $47 \%$ de instituições de natureza pública e $53 \%$ de natureza privada. Conforme se observa no gráfico 1 , do total das faculdades pesquisadas, 96\% utilizam cadáveres humanos nas aulas práticas de Anatomia, enquanto somente 4\% afirmaram não utilizá-los. 
Gráfico 1: Utilização de cadáveres humanos nas Faculdades de Medicina do Brasil, 2015

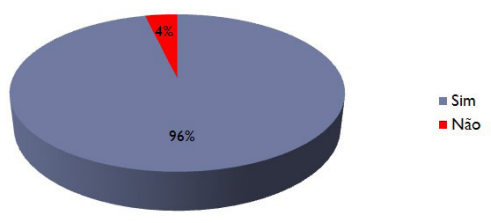

Com relação ao método utilizado na conservação das peças cadavéricas, o Gráfico 2 mostra que 83,3\% utilizam a formolização, 56,4\% a glicerinização e 2,6\% fazem uso da plastinação. O total das respostas soma mais de $100 \%$, pois muitas faculdades utilizam mais de um método de conservação.

Gráfico 2: Método de conservação de cadáver humano utilizado pelas Faculdades de Medicina do Brasil, 2015

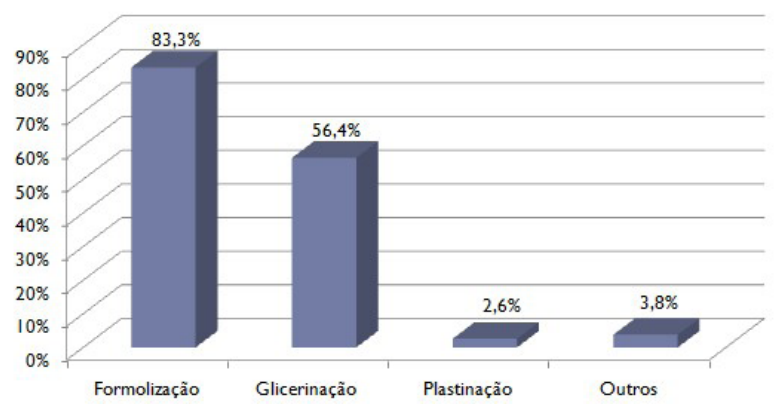

O Gráfico 3 apresenta uma comparação entre a utilização de formol e glicerina na conservação de cadáveres humanos nas cinco regiões brasileiras. Podese constatar que a formolização predomina em todas as regiões, exceto na Centro-Oeste. Observa-se também que a glicerinação tem um maior destaque nas regiões Sul, Sudeste e Centro-Oeste.

Gráfico 3: Uso de formolização e glicerinação pelas Faculdades de Medicina do Brasil por região, 2015

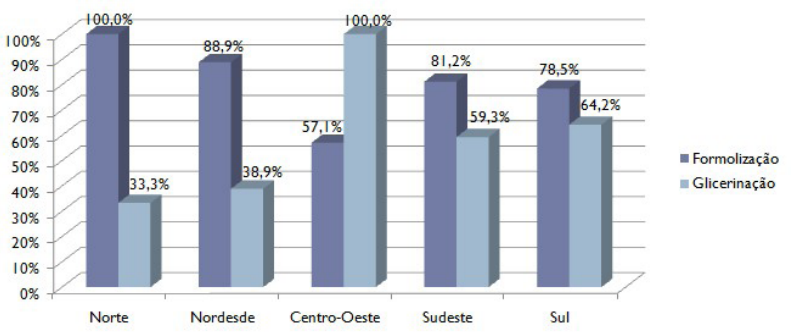

O Gráfico 4 mostra que, dentre as faculdades que utilizam somente o formol na conservação dos cadáveres, $35,3 \%$ pretendem substituir esse método, enquanto $64,7 \%$ não tem essa intenção. Nas instituições que fazem uso de formol e glicerina, $54,8 \%$ pretendem substituir o método de conservação e 45,2\% não pretendem substitui-lo. Por outro lado, nenhuma das instituições que utilizam apenas a glicerina planejam substituir seu método de conservação.

Gráfico 4: Percentual das Faculdades de Medicina do Brasil que pretendem mudar o seu método de conservação de cadáver humano, 2015

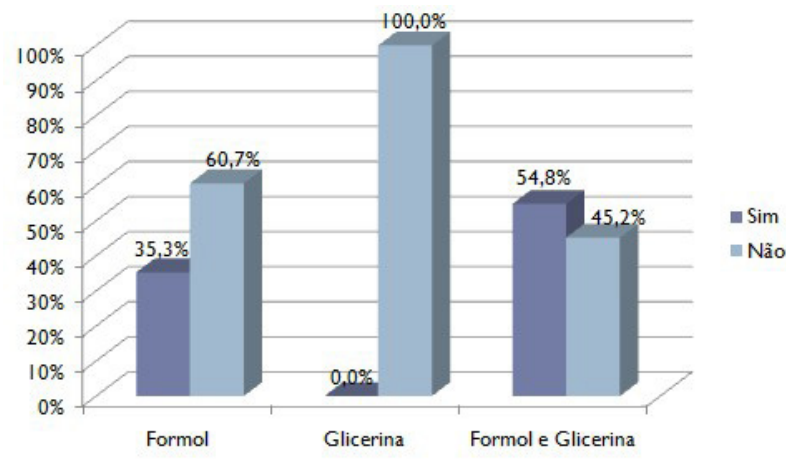

O Gráfico 5 apresenta os motivos pelos quais os docentes desejam mudar o método de conservação de cadáveres. $\mathrm{O}$ principal motivo apontado foi a toxicidade e insalubridade do formol $(70,0 \%)$. Os docentes também destacaram a necessidade de atualização dos métodos $(6,7 \%)$.

Gráfico 5: Motivos pelos quais os docentos desejam mudar o método de conservação de cadáver humano nas Faculdades de Medicina do Brasil, 2015

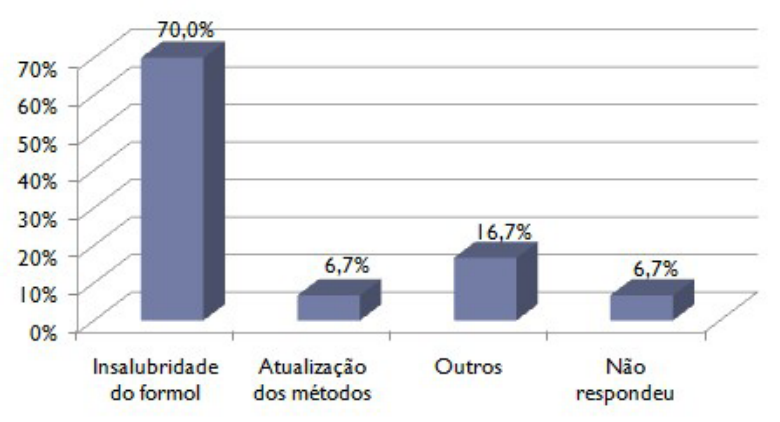

Dentre as instituições que pretendem mudar o método de conservação de cadáveres, $46,7 \%$ responderam que querem substituir o método atual pela glicerinização, $33,3 \%$ por plastinação e 3,3\% ainda estão analisando o método (Gráfico 6).

Quando questionados sobre a satisfação com o método de conservação, dentre os docentes cujas instituições utilizam a formolização, 41,2\% consideraram-se satisfeitos, enquanto que $58,8 \%$, insatisfeitos. Nas instituições que utilizam glicerinização, $54,5 \%$ consideraram-se satisfeitos e $45,5 \%$, insatisfeitos com o método utilizado. Nas instituições que fazem uso concomitante de formolização e glicerinização, $61,3 \%$ dos docentes se consideraram satisfeitos e 38,7\%, insatisfeitos (Gráfico 7). 
Silva, GRS, et al. Métodos de conservação de cadáveres humanos utilizados nas faculdades de medicina.

Gráfico 6: Métodos de conservação de cadáver humano que as Faculdade. de Medicina do Brasil pretendem utilizar futuramente, 2015

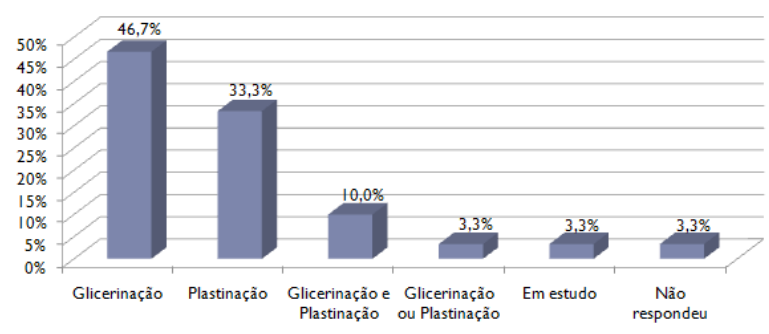

Gráfico 7. Comparação do grau de satisfação dos docentes em relação a utilização de formol e glicerina nas Faculdades de Medicina do Brasil, 2105

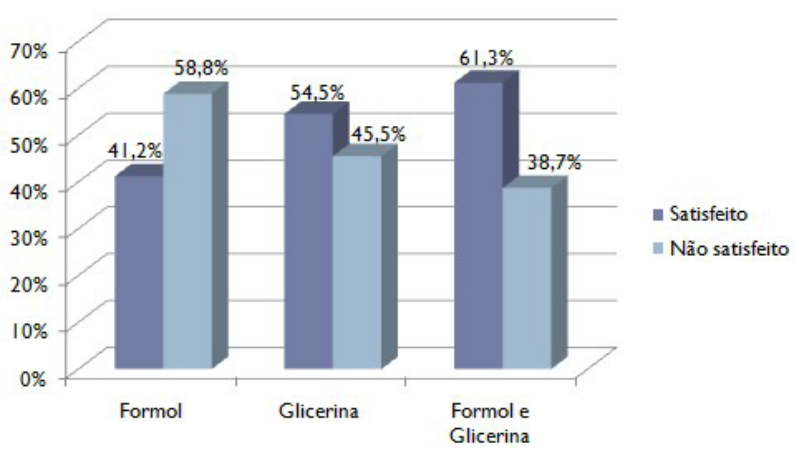

\section{DISCUSSÃO}

A aprendizagem é um processo que depende da interação comportamental entre professores e alunos, influenciada pelos recursos didáticos-pedagógicos que os docentes adotam para motivar seus alunos a desenvolver o conhecimento ${ }^{13,14}$.

Os avanços científicos e tecnológicos ocorridos em grande escala durante as últimas décadas, acarretaram inúmeras mudanças na estrutura da sociedade. Essas mudanças foram sentidas no próprio meio acadêmico, de modo que todos se depararam com as inovações técnicas e com a nova maneira de lidar com a ciência em seus vários ramos. Entretanto, acredita-se que o recurso didático mais utilizado atualmente para o ensino prático da Anatomia Humana nas Faculdades de Medicina do Brasil é o mesmo desde 500 anos antes de Cristo. A tecnologia ainda não foi capaz de substituir com fidedignidade as estruturas humanas apresentadas pelo cadáver, já que $96 \%$ das instituições de ensino médico adotam o cadáver humano como o principal recurso didático-pedagógico nas aulas de anatomia.

Acredita-se que há um consenso entre docentes e acadêmicos sobre o uso dos cadáveres humano de ensino da anatomia. Estudos demonstraram que $88,9 \%$ dos alunos dos cursos do Centro de Saúde da Universidade Federal de
Pernambuco consideram indispensável o uso de cadáver humano nas aulas práticas. No entanto, o uso desse recurso está prejudicado devido a redução do número de cadáveres doados e a falta de conhecimento e conscientização da sociedade sobre os aspectos éticos e legais da doação dos $\operatorname{corpos}^{15}$.

O uso de cadáveres para fins de ensino e pesquida é regularizado de acordo com a Lei 8501/92. Esta Lei prever que cadáver não reclamado junto às autoridades pública, no prazo de 30 dias, poderá ser destinado às escolas de medicina, para fins de estudo e pesquisa de caráter científico. O Código Civil brasileiro relata que após a morte diversos direitos de personalidade são perdidos, porém o único mantido é o direito à honra, umbilicalmente associado à natureza humana. Dessa forma, o respeito ao cadáver deve ser mantido, algo que é reforçado pela famosa "Oração ao Cadáver Desconhecido”, de autoria de Karl Rokitansky ${ }^{16,17}$.

Os docentes possuem a função de conscientizar os acadêmicos sobre o respeito perante o cadáver, pois aquele corpo antes da morte construiu uma história influenciada por vários sentimentos humanos e que agora continua o seu legado servindo a sociedade de uma outra maneira, algo que deve ser honrado.

Diante da dificuldade na aquisição de novos cadáveres pelas Faculdades de Medicina no Brasil, a solução adotada de imediato é adoção de métodos eficazes de conservação de cadáveres. Constatou-se que o principal método de conservação adotado pelas Faculdades de Medicina no Brasil é a formolização. Isso se deve ao fato desse método ter um custo menor e conservar por um tempo bem prolongado, ou seja, um bom custo e benefício. Entretando, possui uma alta toxicidade, que provoca irritação no bulbo ocular, nas vias aéreas superiores, desconforto respiratório e efeito carcinogênico ${ }^{18}$.

Os dados obtidos evidenciaram que o uso do formol como fixador é predominante nas instituições de todas as regiões do Brasil, exceto na Centro-Oeste. Porém acredita-se que esse resultado pode ter sido influenciado pelo número menor de respostas proveniente dessa região em comparação as demais, visto que enquanto na região sudeste foi recebido trinta e uma respostas, na região centrooeste esse número foi apenas de oito.

Atualmente, sabe-se que o fator econômico é de suma importância em qualquer aspecto que caracteriza uma sociedade, e com o ensino da Anatomia Humana não é diferente. Acredita-se que o gráfico 3 pode retratar a desigualdade econômica vingente no território brasileiro. As diferenças na distribuição dos recursos científicos e tecnológicos são também muito acentuadas. Parte dos grupos atuantes em pesquisas se encontram principalmente nas Regiões Sul e Sudeste, onde há maior investimento na área de ciência e tecnologia. Assim, a glicerinação, por ser um método de custo mais elevado, tem um maior destaque no Sudeste e Sul do país quando comparado com o Norte e Nordeste, regiões de menor poder econômico ${ }^{19}$. 
Silva, GRS, et al. Métodos de conservação de cadáveres humanos utilizados nas faculdades de medicina.

O método de glicerinação apesar do alto custo, todas as faculdades que a utilizam, não planejam mudar tal processo de conservação. Sabe-se que as peças glicerizadas são mais fáceis de se manusear e apresentam menor intensidade de peso e cheiro, devido à diminuição de vapores prejudiciais aos manipuladores e excelentes resultados estéticos e morfológicos. Já nas intituições que utilizam o formol, mesmo com as suas desvantagens, 60,7\% não planejam alterar o método. Acredita-se que isso se deve ao fato do formaldeído ainda possuir algumas qualidades, tais como baixo custo, rápida penetração e adequada conservação por longo período ${ }^{7,20}$.

Entretanto, uma quantidade considerável dos docentes das faculdades que utilizam a formolização estão insatisfeitos e uma boa parte pretende mudar tal método de conservação, por conta da insalubridade do formol, pois o uso do formaldeído é prejudicial a saúde, em que os principais afetados são os docentes, pesquisadores e técnicos de laboratórios, ou seja, aqueles que estão em contato por longos períodos ${ }^{21}$.

\section{REFERÊNCIAS}

1. Freitas V. Anatomia: conceitos e fundamentos. Porto Alegre: Artmed; 2004.

2. Corbin A, Courtine J, Vigarello G. História do corpo - da renascença às luzes. Petrópolis: Vozes; 2008.

3. Nova JLL, Bezerra Filho JJ, Bastos LA. Lição de anatomia. Interface (Botucatu). 2000;4(6):87-96. http://dx.doi. org/10.1590/S1414-32832000000100007.

4. Rodrigues H. Técnicas anatômicas. 3a ed. Vitória; 2005.

5. Sant'ana APF. Utilização do formaldeído em diferentes concentrações associado ou não ao cloreto de sódio na conservação de tecidos de ovinos. In: Anais do Conbravet; 2008. p. 1277.

6. Tavano PT, Oliveira MC. Surgimento e desenvolvimento da ciência anatômica. Anuário Prod Acad Docente. 2009;2(3):7384. Disponível em: http://sare.anhanguera.com/index.php/ anudo/article/view/683.

7. Silva R, Maria-do-Ó C, Brito V, Oliveira B, Costa É, Moura $\mathrm{G}$. Vantagens e desvantagens das técnicas de preparação de materiais didáticos para as aulas práticas de morfologia. Rev Didática Sistêmica. 2011;13(2):24-41. Disponível em: https:// www.seer.furg.br/redsis/article/view/2237.

8. Babinski MA, Sgrott EA, Luz HP, Brasil FB, Chagas MA, Abidu-Figueiredo M. La relacion de los estudiantes com el cadáver em el estudo práctico de anatomia: la reaccioón e influencia em el aprendizaje. Int J Morphol.. 2003;21(2):13742. http://dx.doi.org/10.4067/S0717-95022003000200007.

9. Rizzolo LJ, Stewart WB. Should we continue teaching anatomy by dissection when...?. Anat Rec. 2006;289:215-8. doi: 10.1002/ar.b.20117.

10. Anyanwu GE, Udemezue, OO, Obikili EN. Dark age of sourcing cadavers in developing countries: a Nigerian survey. Clin Anat. 2011;24:831-6. doi: 10.1002/ca.21187.
Para escolher a melhor forma de conservação das peças anatômicas, vários fatores são analisados: os custos, a toxicidade, a técnica, o manuseio das peças no pós-preparo, a necessidade de manutenção da morfologia e coloração a mais próxima possível do estado real e o odor. Assim, por conta da insalubridade do formol, o principal método almejado pelas faculdades, que planejam alterar a metodologia de conservação, é a glicerinação ${ }^{22}$.

\section{CONCLUSÃO}

Através dos resultados obtidos na presente pesquisa, conclui-se que a grande maioria das faculdades brasileiras de Medicina utlizam cadáveres humanos no ensino da Anatomia Humana. O método mais utilizado na conservação das peças cadavéricas ainda é a formolização, seguido da glicerinização. Porém parte das instituições que utilizam o formaldeído desejam substituir esse método, em virtude, principalmente, da sua insalubridade, sendo a glicerinação o método mais almejado.

11. Collipal LE, Silva MH. Estudio de la anatomía en cadáver y modelos anatómicos: impresión de los estudiantes. Int J Morphol. 2011;29(4):1181-5. http://dx.doi.org/10.4067/ S0717-95022011000400018.

12. Kerby J, Shukur ZN, Shalhoub J. The relationships between learning outcomes and methods of teaching anatomy as perceived by medical students. Clin Anat. 2011;24:489-97. doi: $10.1002 / \mathrm{ca} .21059$.

13. Castoldi R, Polinarski CA. A utilização de recursos didáticopedagógicos na motivação da aprendizagem. In: I Simpósio Nacional de Ensino de Ciência e Tecnologia. Curitiba, Paraná: Universidade Tecnológica Federal do Panará; 2009. p.684-92.

14. ubo $\mathrm{O}$, Botomé S. Ensino-aprendizagem: uma interação entre dois processos comportamentais. Interação Psicol. 2001;5(1). http://dx.doi.org/10.5380/psi.v5i1.3321.

15. Costa GBF, Costa GBF, Lins CCSA. O cadáver no ensino da anatomia humana: uma visão metodológica e bioética. Rev Bras Educ Med. 2012;36(3):369-73. https://dx.doi. org/10.1590/S0100-55022012000500011.

16. Queiroz CAF. O uso de cadáveres humanos como instrumento na construção de uma visão bioética [mestrado]. Goiás: Universidade Católica de Goiás; 2005. Disponível em: http:// tede2.pucgoias.edu.br:8080/bitstream/tede/3106/1/Carla $\% 20$ de\%20Alcantara\%20Ferreira\%20Queiroz.pdf.

17. Gagliano PS. Novo curso de direito civil. São Paulo: Saraiva; 2006.

18. Sousa AM, Carvalho LD, Maia LS, Soares RMO, Audi SG. Formaldeído: grau de percepção de professores e técnicos de laboratórios universitários de anatomia, quanto aos riscos ocupacionais e uso de EPI'S. FIEP Bull Online. 2015;85(1). doi: $10.16887 / 85 . a 2.93$.

19. De Barros FAF Os desequilíbrios regionais da produção técnico-ciêntifica. São Paulo Perspectiva. 2000;14(3):12-9. 
Silva, GRS, et al. Métodos de conservação de cadáveres humanos utilizados nas faculdades de medicina.

https://dx.doi.org/10.1590/S0102-88392000000300004.

20. Kimura AK, Carvalho WL. Estudo da relação custo-benefício no emprego da técnica de glicerinização em comparação com a utilização por formol [Tese]. Araraquara: Universidade Estadual Paulista; 2010.

21. Viera IIF Dantas BPA Ferreira FCM Carvalho RBAC Carvalho Freire IBF Neto EJS. Efeitos da utilização do formaldeído em laboratórios de anatomia. Re. Ciênc Saúde
Nova Esperança (São Paulo). 2013;11(1):97-105.

22. Silva EM, Souza Filho GD, Souza MCT, Marques t, Furtado JM. Estudo analítico da Técnica de Glicerinização empregada para conservação de peças anatômicas: experiência da disciplina de Anatomia Humana do Departamento de Morfologia da UniFOA. Cad UniFOA (Rio de Janeiro). 2008; ed.esp.:66-9. Disponível em: http://web.unifoa.edu.br/ cadernos/especiais/pos-graduacao/01/66.pdf. 